10

\title{
О возможности применения фторидных неодим-активированных кристаллов в качестве светоуправляемого затвора для излучения ArF-лазера (193 nm)
}

\author{
() Р.Д. Аглямов, А.К. Наумов, А.В. Ловчев, О.А. Морозов \\ Университет КФУ, \\ 420008 Казань, Россия \\ e-mail: aglyam92@mail.ru
}

Поступила в редакцию 01.12.2020 г.

В окончательной редакции 01.12.2020 г.

Принята к публикации 15.12.2020 г.

\begin{abstract}
Сообщено об оптических свойствах кристаллов фторидов $\mathrm{KY}_{3} \mathrm{~F}_{10}: \mathrm{Nd}^{3+}, \mathrm{Na}_{3} \mathrm{Y}_{6} \mathrm{~F}_{22}: \mathrm{Nd}^{3+}, \mathrm{SrF}_{2}: \mathrm{Nd}^{3+}$ и $\mathrm{CaF}_{2}: \mathrm{Nd}^{3+}$ в УФ и ВУФ областях спектра. Спектры поглощения из основного $\left({ }^{4} I_{9 / 2}\right)$ и из возбужденного $\left({ }^{4} F_{3 / 2}\right)$ состояний зарегистрированы в диапазоне длин волн от 190 до $240 \mathrm{~nm}$. Спектры поглощения из возбужденного состояния показывают существенное приращение коэффициентов поглощения исследованных образцов в области длин волн $193 \mathrm{~nm}$ при накачке состояния ${ }^{4} F_{3 / 2}$ ионов $\mathrm{Nd}^{3+}$ ИК излучением. Полученные результаты демонстрируют возможность применения этих кристаллов в качестве оптических затворов для УФ и ВУФ диапазона, управляемых внешним лазерным ИК излучением, например для излучения эксимерного ArF-лазера с длиной волны $193 \mathrm{~nm}$, которое начало применяться в фотолитографии.
\end{abstract}

Ключевые слова: фторидный кристалл, ионы неодима, ВУФ, УФ, спектр поглощения, поглощение из возбужденного состояния, оптический затвор, $193 \mathrm{~nm}$, эксимерный ArF-лазер.

DOI: $10.21883 /$ OS.2021.04.50784.293-20

\section{Введение}

Изучение спектроскопических свойств активированных фторидных кристаллов в УФ и ВУФ областях спектра, обусловленных межконфигурационными $4 f \leftrightarrow 4 f 5 d$-переходами ионов редкоземельных элементов (РЗЭ), ведутся довольно давно [1,2]. Так, в работах $[3,4]$ представлены как теоретические расчеты спектров межконфигурационных переходов, так и их экспериментальное подтверждение. Все эти исследования важны для обогащения знаний о спектроскопических свойствах этих кристаллов в УФ и ВУФ областях спектра, а также для возможности использования последних в качестве активных сред лазеров и других возможных практических применений.

При изучении спектроскопических свойств этих кристаллов в УФ и ВУФ областях спектра было обнаружено явление поглощения из возбужденного состояния (ПВС) на смешанные состояния $\left({ }^{4} F_{3 / 2} \rightarrow 4 f^{2} 5 d^{1}\right)$ ионов $\mathrm{Nd}^{3+}$ [5]. В некоторых публикациях ПВС в активированных кристаллах исследователями рассматривается негативным явлением для их применения в качестве активных сред [6,7]. В данной работе показан пример возможного практического применения явления ПВС.

Лазеры УФ и ВУФ диапазона широко используются в спектроскопии, мониторинге окружающей среды, медицине, фотолитографии и т. п. В связи с этим актуальна разработка и усовершенствование методов управления их излучением. Оптические затворы УФ диапазона представляют значительный интерес для целого ряда приложений. Например, в фотолитографии [8], в технологии лазерной абляции [9] и в некоторых других областях медицины используются эксимерные ArF-лазеры с длиной волны излучения $193 \mathrm{~nm}$ [10]. Для управления их излучением зачастую применяются механические затворы. Однако эти затворы малопригодны для управления излучением из-за большого времени срабатывания, поэтому они применяются лишь в качестве заслонок. В этой работе в экспериментах по исследованию явления ПВС во фторидных кристаллах, активированных ионами трехвалентного неодима, показана возможность его использования как механизма оптических затворов для излучения с длиной волны $193 \mathrm{~nm}$, управляемых внешним лазерным излучением ближнего ИК диапазона спектра.

Идея светоуправляемого оптического затвора (СУОЗ) впервые была предложена и экспериментально продемонстрирована в работе [11]. Данный затвор, работа которого основана на использовании $f-f$-переходов ионов $\mathrm{Nd}^{3+}$, позволял „включать и выключать“ излучение в спектральной области 260-320 nm. Но, видимо, по причине того, что потребность в практическом использовании этого спектрального диапазона отсутствовала, идея не получила развития.

\section{Принцип действия СУОЗ. Критерии отбора объектов исследований}

Принцип действия СУОЗ представлен на обобщенной диаграмме энергетических состояний ионов $\mathrm{Nd}^{3+}$ в кристаллах (рис. 1). Исходно система находится в состо- 


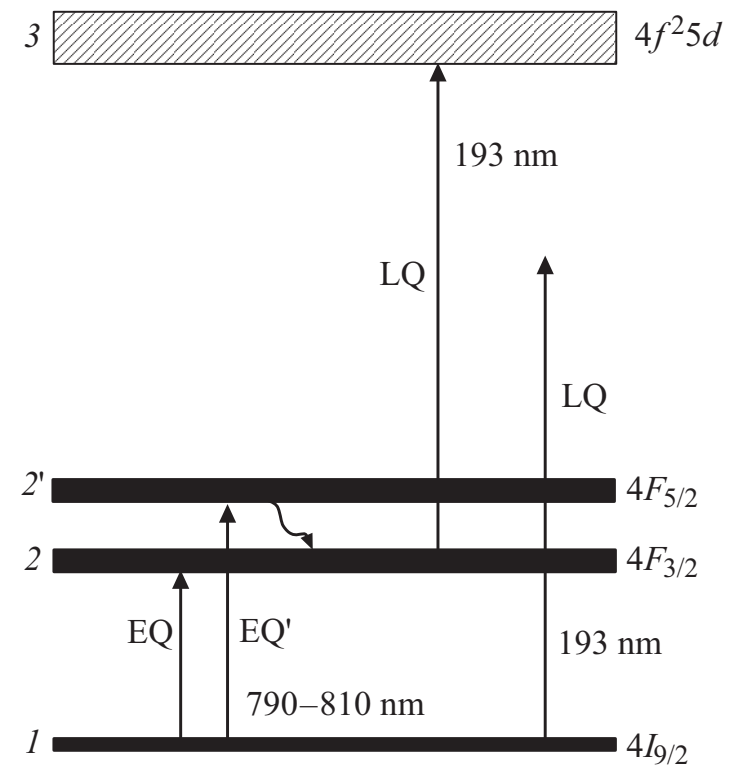

Рис. 1. Обобщенная диаграмма энергетических состояний ионов $\mathrm{Nd}^{3+}$ с переходами, демонстрирующими принцип действия СУОЗ.

янии 1. Запираемые кванты (LQ) в этом состоянии не поглощаются в связи с тем, что вверху отсутствуют состояния, положение которых равно энергии квантов LQ. Поэтому кванты LQ проходят сквозь образец кристалла без поглощения. Если создать населенность состояния 2 квантами возбуждения (EQ) напрямую (резонансно) или через промежуточное состояние $2^{\prime}$ с последующим безызлучательным электронным переходом на состояние 2, то с этого возбужденного состояния 2 энергии квантов LQ будет достаточно для достижения верхнего состояния 3 смешанной конфигурации $4 f^{2} 5 d^{1}$ ионов $\mathrm{Nd}^{3+}$, и кванты LQ будут поглощаться. В результате накачанная среда СУO3 перестанет пропускать кванты LQ, т.е. затвор закроется. Надо отметить, что состояние 2 ионов $\mathrm{Nd}^{3+}$ метастабильное и эффективно накапливает электроны при поглощении квантов возбуждения (EQ или $\left.\mathrm{EQ}^{\prime}\right)$.

Диаграмма на рис. 1 приведена для случая использования в кристаллах ионов $\mathrm{Nd}^{3+}$, однако описанную схему можно реализовать на переходах других редкоземельных ионов. Тем не менее для реализации СУОЗ использование ионов $\mathrm{Nd}^{3+}$ на настоящий момент является наиболее благоприятным. Состояние ${ }^{4} F_{3 / 2}$ в СУОЗ используется в качестве состояни 2. Это состояние метастабильное и обычно используется как верхний лазерный уровень для микронных $\mathrm{Nd}$-лазеров. В настоящее время для накачки этого состояния разработан большой ассортимент диодных лазеров с широкой линейкой мощностей и конфигураций выходного излучения.

При реализации СУОЗ для квантов с длиной волны 193 nm использование фторидных кристаллов принципиально необходимо, так как в этом диапазоне граница их прозрачности лежит в области $120-150 \mathrm{~nm}$, в то время как у оксидных кристаллов этот предел составляет 250-350 nm [12,13].

В качестве состояния 3 для реализации СУОЗ, как упоминалось выше, возможно использование состояний смешанной $4 f^{2} 5 d^{1}$-конфигурации ионов $\mathrm{Nd}^{3+}$. Эти состояния по шкале энергий расположены от основного состояния на расстоянии, равном сумме энергий кванта LQ и кванта EQ, равной энергии состояния 2. Относительные положения состояний $4 f$ (конфигурации трехвалентных ионов РЗЭ) от кристалла к кристаллу имеют разброс по энергии не более чем $50-400 \mathrm{~cm}^{-1}$ [14]. Состояние ${ }^{4} F_{3 / 2}$ ионов $\mathrm{Nd}^{3+}$ в кристалле $\mathrm{LaF}_{3}$ расположено по энергии на уровне $11600 \mathrm{~cm}^{-1}$ [15]. Значение энергии запираемого кванта (LQ - $193 \mathrm{~nm})$ составляет около $51800 \mathrm{~cm}^{-1}$. Таким образом, $\mathrm{Nd}^{3+}$ активированный кристалл-кандидат для СУОЗ на $193 \mathrm{~nm}$ должен иметь положение состояний $4 f^{2} 5 d^{1}$ по шкале энергий на уровне около $63500 \mathrm{~cm}^{-1}$. Иными словами, этот кристалл должен иметь полосу поглощения в области $157 \mathrm{~nm}$. Причем поглощение из основного состояния ионов $\mathrm{Nd}^{3+}$ в этом кристалле в области $193 \mathrm{~nm}$ (энергия кванта равна $51800 \mathrm{~cm}^{-1}$ ) в идеальном случае должно отсутствовать.

В соответствии с отмеченными выше требованиями для применения в качестве СУОЗ ВУФ диапазона, используя данные литературы [16-20], были отобраны такие фторидные $\mathrm{Nd}$-активированные кристаллы, у которых верхняя граница окна прозрачности лежит в области 120-150 nm и которые обладают простой кубической структурой. Последнее исключает проблемы как непосредственно при проведении исследований, так и при возможном их практическом применении, связанные с поляризационными эффектами, возникающими с кристаллами, обладающими оптической осью. Также в перспективе (при использовании СУОЗ на практике) кристалл должен быть технологичным: иметь недорогие компоненты, простую и низко затратную технологию выращивания. Еще одним критерием при выборе объектов для СУОЗ была низкая начальная интенсивность полос поглощения ионов $\mathrm{Nd}^{3+}$ в спектральной области от 180 до $220 \mathrm{~nm}$. Этот выбор осуществлялся по спектроскопическим данным из серии работ [2-6,16-20].

\section{Образцы и схема экспериментальной установки}

Для нашей работы были отобраны кристаллы $\mathrm{KY}_{3} \mathrm{~F}_{10}(\mathrm{KYF}): \mathrm{Nd}^{3+}, \mathrm{Na}_{3} \mathrm{Y}_{6} \mathrm{~F}_{22}(\mathrm{NYF}): \mathrm{Nd}^{3+}$ и $\mathrm{SrF}_{2}: \mathrm{Nd}^{3+}$, $\mathrm{CaF}_{2}: \mathrm{Nd}^{3+}$, концентрация ионов $\mathrm{Nd}^{3+}$ в образцах задавалась 1.5 at.\%.

Образцы этих отобранных кристаллов были выращены в научно-исследовательской лаборатории магнитной радиоспектроскопии и квантовой электроники института физики Казанского федерального университета методом Бриджмена во фторированной атмосфере аргона. Полученные кристаллы имели форму були диаметром 5 и 


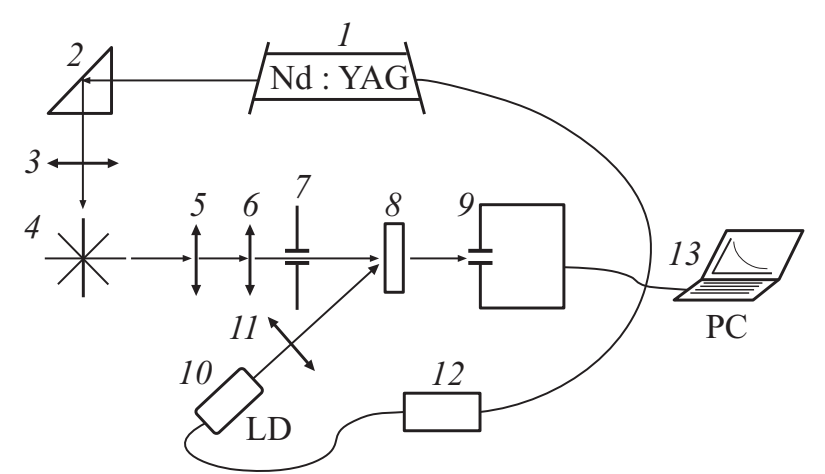

Рис. 2. Схема экспериментальной установки „накачказондирование“. 1 - YAG:Nd-лазер; 2 - призма; 3 - собирающая линза; 4 - лазерная искра; 5, 6 - телескоп Галилея; 7 - диафрагма; 8 - исследуемый образец; 9 - спектрометр; 10 - диодный лазер; 11 - собирающая линза; 12 - блок синхронизации; 13 - компьютер.

длиной $30 \mathrm{~mm}$, из которых были подготовлены образцы для оптических экспериментов. Эти образцы имели форму дисков толщиной $2 \mathrm{~mm}$ с плоскопараллельными полированными сторонами.

Схема экспериментальной установки приведена на рис. 2. В канале зондирования использовалась лазерная искра (ЛИ) в воздухе, возбуждаемая излучением лазера на иттрий-алюминиевом гранате. В канале накачки использовался диодный лазер, у которого была возможность перестройки длины волны выходного излучения для точной подстройки в максимумы полос поглощения исследуемых образцов.

Во всех экспериментах для уменьшения тепловой нагрузки на образцы применялся импульсный режим накачки. В канале накачки использовался диодный лазер с длительностью импульсов излучения $1 \mathrm{~ms}$, что было заведомо больше времени жизни состояния ${ }^{4} F_{3 / 2}$ ионов $\mathrm{Nd}^{3+}$ в большинстве фторидных кристаллов. Импульсы накачки диодного лазера синхронизировались с импульсами генерации Nd:YAG-лазера, который применялся для возбуждения ЛИ. Частота следования импульсов $\mathrm{Nd}$ :YAG-лазера составляла $3 \mathrm{~Hz}$, их длительность $7 \mathrm{~ns}$. Регистрация спектров осуществлялась спектрометром на основе монохроматора МУМ-2, оборудованного ПЗСлинейкой. Управление экспериментом, регистрация результатов измерений и их усреднение осуществлялись с помощью компьютера.

\section{Результаты и обсуждение}

В результате экспериментов были получены спектры поглощения образцов кристаллов $\mathrm{KYF}_{\mathrm{Nd}}{ }^{3+}, \mathrm{NYF}: \mathrm{Nd}^{3+}$, $\mathrm{SrF}_{2}: \mathrm{Nd}^{3+}$ и $\mathrm{CaF}_{2}: \mathrm{Nd}^{3+}$ как при воздействии излучения накачки (спектры поглощения из возбужденного состояния), так и без него (спектры из основного состояния). Эти спектры, зарегистрированные в спектральной области $190-240 \mathrm{~nm}$, представлены на рис. 3. Цифрой 1 на рисунках обозначены спектры поглощения образцов при накачке состояний ${ }^{4} F_{3 / 2}$ (через состояния ${ }^{4} F_{5 / 2}$ $\left.\left({ }^{2} H_{9 / 2}\right)\right)$. Спектры поглощения образцов из основного состояния ${ }^{4} I_{9 / 2}$ ионов $\mathrm{Nd}^{3+}$ обозначены цифрой 2 .

Как видно из рисунка, в спектрах при воздействии излучения накачки с оценочной плотностью $3-5 \mathrm{~kW} / \mathrm{cm}^{2}$ для всех образцов наблюдалось существенное приращение поглощения.

На рис. 3 отрезком $\Delta$ со стрелками показана разница в интенсивности поглощения накачанных и ненакачанных образцов на длине волны $193 \mathrm{~nm}$.

Наибольшее приращение интенсивности поглощения обнаружилось у образца $\mathrm{CaF}_{2}: \mathrm{Nd}^{3+}$ (рис. $3, a$ ), которое составило около $2.55 \mathrm{~cm}^{-1}$.

Как видно из рис. $3, a-d$, у исследованных здесь кристаллов изменения коэффициентов поглощения при накачке начинаются с длин волн $220-230 \mathrm{~nm}$ и возрастают при продвижении в коротковолновую область, причем с различной интенсивностью. Так, наименьшее приращение поглощения наблюдается у образца KYF: $\mathrm{Nd}^{3+}$ (рис. $3, c$ ), которое, начиная с $230 \mathrm{~nm}$, достигает максимума со значением в $1.2 \mathrm{~cm}^{-1}$ при длине волны $197 \mathrm{~nm}$. Далее при продвижении в коротковолновую сторону спектра интенсивность поглощения у этого кристалла уменьшается. В этом кристалле, по всей видимости, непростая структура уровней смешанной конфигурации $4 f^{2} 5 d^{1}$ ионов $\mathrm{Nd}^{3+}$, у которой в области $155 \mathrm{~nm}$ имеется „провал“ спектре поглощения [2]. Этот „провал“ проявляется в том, что в области $193 \mathrm{~nm}$ образец кристалла KYF: $\mathrm{Nd}^{3+}$ при накачке состояния ${ }^{4} F_{3 / 2}$ ионов $\mathrm{Nd}^{3+}$ поглощает всего лишь с коэффициентом поглощения $0.83 \mathrm{~cm}^{-1}$.

У образцов кристаллов $\mathrm{SrF}_{2}: \mathrm{Nd}^{3+}$ и $\mathrm{NYF}: \mathrm{Nd}^{3+}$ (рис. $3, b$ и $d$ соответственно) приращение коэффициентов поглощения также наблюдается, начиная с $230 \mathrm{~nm}$. При продвижении в коротковолновую сторону интенсивность приращения поглощения, судя по спектрам этих кристаллов, в области около $200 \mathrm{~nm}$ выходят на постоянное значение. При этом у образца кристалла NYF: $\mathrm{Nd}^{3+}$ приращение на $193 \mathrm{~nm}$ составляет $2.1 \mathrm{~cm}^{-1}$, у $\mathrm{SrF}_{2}: \mathrm{Nd}^{3+}$ - около $1.45 \mathrm{~cm}^{-1}$. При этом доля поглощенной мощности накачки каждым из образцов составляла около 95 и $42 \%$ соответственно.

Проведенные эксперименты показали, что приращение поглощения при возбуждении состояния ${ }^{4} F_{3} / 2$ ионов $\mathrm{Nd}^{3+}$ наблюдается у всех исследованных образцах. Как видно из рис. $3, a$, лучший результат показал образец кристалла $\mathrm{CaF}_{2}: \mathrm{Nd}^{3+}$, у которого приращение интенсивности поглощения при оценочной плотности возбуждения около $3-5 \mathrm{~kW} / \mathrm{cm}^{2}$ составило $2.55 \mathrm{~cm}^{-1}$ при том, что в образце поглощалось всего $65 \%$ мощности накачки.

Такое поглощение областью в кристаллах, накачанной внешним лазерным излучением, может существенно ослабить излучение с длинами волн около 190-205 nm. Это свойство можно использовать для управления отмеченной спектральной областью, в частности областью около $193 \mathrm{~nm}$, т. е. запирать ее. 

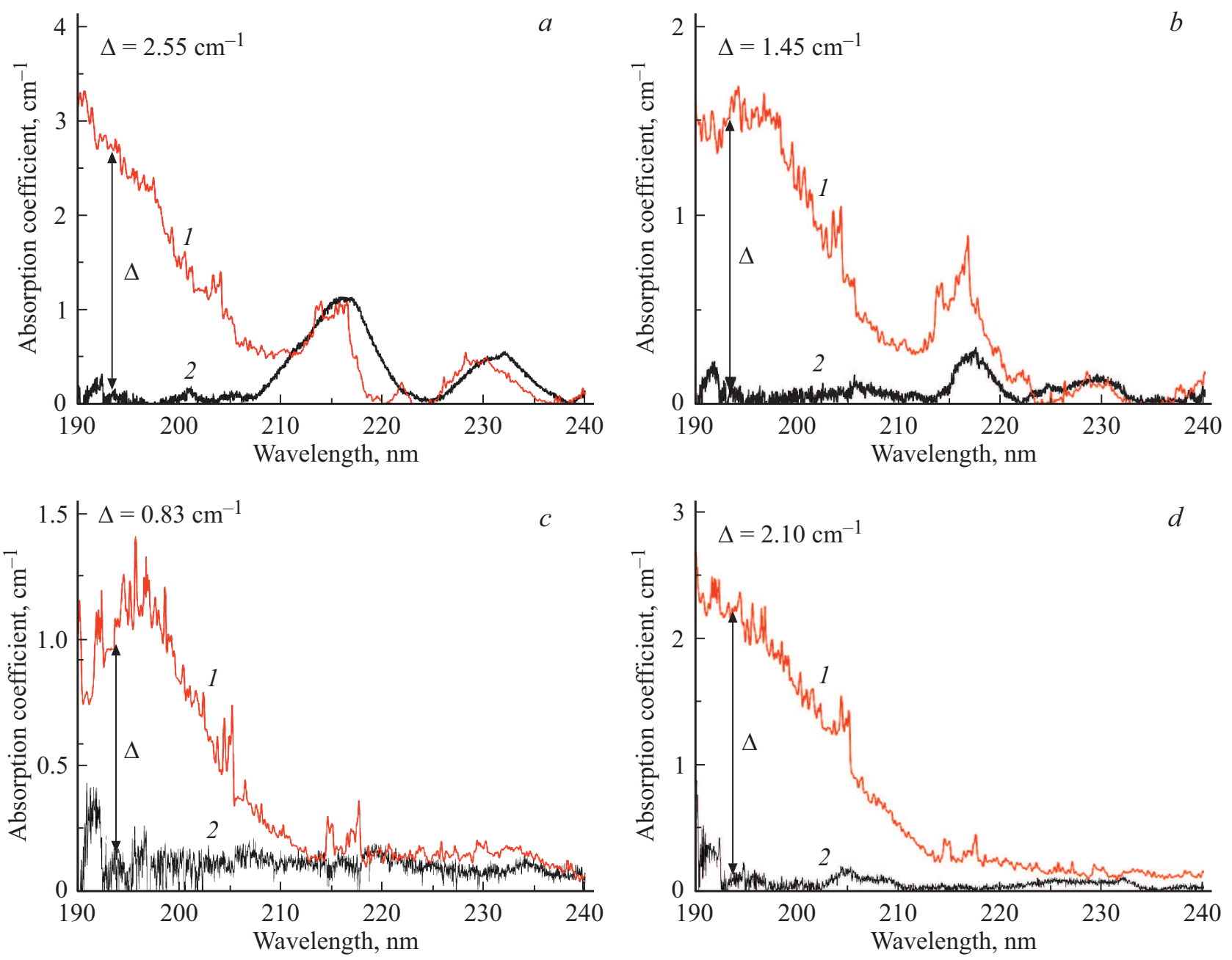

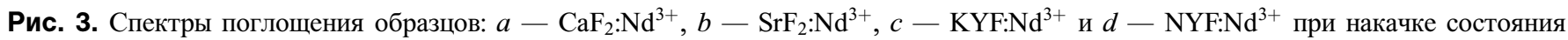
${ }^{4} F_{3 / 2}(1)$ и без накачки (2). $\Delta$ - приращение поглощения образцов на длине волны $193 \mathrm{~nm}$.

\section{Заключение}

В результате проведенных экспериментов получены данные по спектрам поглощения из возбужденного ${ }^{4} F_{3 / 2}$ состояния кристаллов $\mathrm{KYF}: \mathrm{Nd}^{3+}, \mathrm{NYF}: \mathrm{Nd}^{3+}, \mathrm{SrF}_{2}: \mathrm{Nd}^{3+}$ и $\mathrm{CaF}_{2}: \mathrm{Nd}^{3+}$ в спектральной области $190-240 \mathrm{~nm} \mathrm{c}$ использованием источника сплошного спектра - ЛИ в воздухе. Общий вид полученных спектров хорошо согласуется с данными по кристаллу NYF:Nd из работы [21]. На полученных спектрах продемонстрирована возможность использования фторидных кристаллов, активированных ионами трехвалентного неодима, в качестве оптических затворов для лазерного излучения с длиной волны $193 \mathrm{~nm}$ с управлением внешним лазерным излучением ближнего ИК диапазона. Результаты проведенных исследований показывают, что кристаллы монофторидов $\mathrm{SrF}_{2}$ и $\mathrm{CaF}_{2}$ (в сравнении с двойными кристаллами KYF, NYF), активированные ионами $\mathrm{Nd}^{3+}$, наиболее перспективны для применения в качестве СУОЗ. Кристалл $\mathrm{CaF}_{2}$ (хорошо всесторонне изученный, с отработанной технологией выращивания образцов больших размеров - их выращивают до $300 \mathrm{~mm}$ в диаметре [22]), активированный ионами $\mathrm{Nd}^{3+}$, показал наиболее высокие характеристики приращения поглощения при накачке ${ }^{4} F_{3 / 2}$-состояния. К тому же отметим, что чистый монокристалл $\mathrm{CaF}_{2}$ при использовании ArFлазера может применяться в фотолитографии как материал для линз. Этот материал - прозрачный в ВУФ и стойкий к воздействию лазерного излучения $193 \mathrm{~nm}$.

\section{Финансирование работы}

Работа выполнена за счет средств субсидии, выделенной Казанскому федеральному университету для выполнения государственного задания в сфере научной деятельности № 0671-2020-0050.

\section{Конфликт интересов}

Авторы заявляют, что у них нет конфликта интересов. 


\section{Список литературы}

[1] Dubinskii M.A., Cefalas A.C., Sarantopoulou E., Abdulsabirov R.Yu., Korableva S.L., Naumov A.K., Semashko V.V.// Opt. Commun. 1992. V. 94. N 1-3. P. 115. doi 10.1016/00304018(92)90417-P

[2] Kolia Z., Sarantopoulou E., Cefalas A.C., Nicolaides C.A., Naumov A.K., Semashko V.V., Abdulsabirov R.Yu., Korableva S.L., Dubinskii M.A. // JOSA B. 1995. V. 12. N 5. P. 782. doi 10.1364/JOSAB.12.000782

[3] Van Pieterson L., Reid M.F., Wegh R.T., Soverna S., Meijerink A. // Phys. Rev. B. 2002. V. 65. N 4. P. 451131.

[4] Peijzel P.S., Vergeer P., Meijerink A., Reid M.F., Boatner L.A., Burdick G.W. // Phys. Rev. B. 2005. V. 71. P. 045116. doi 10.1103/PhysRevB.71.045116

[5] Guyot Y., Guy S., Joubert M.F. // J. Alloys Compd. 2001. V. 323-324. P. 722. doi 10.1016/S0925-8388(01)01059-3

[6] Lawson J.K., Payne S.A. // JOSA B. 1991. V. 8. N 7. P. 1404. doi 10.1364/JOSAB.8.001404

[7] Fornasiero L., Kellner T., Kück S., Meyn J.P., Möbert P.E.A., Huber G. // Appl. Phys. B. 1999. V. 68. N 1. P. 67. doi $10.1007 / \mathrm{s} 003400050587$

[8] Lindner R., Stamm U., Patzel R., Basting D. // Microelectronic Engineering. 1998. V. 41-42. P. 75. doi 10.1016/S01679317(98)00016-1

[9] Aesa A.A., Walton C.D. // Appl. Phys. A. Mater. Sci. Process. 2018. V. 124. N 6. P. 465. doi 10.1007/s00339-018-1859-Z

[10] Blum S.E., Srinivasan R., Wynne J.J., Kisco Mt. Patent US4784135, 1986.

[11] Dubinskii M.A. // J. Mod. Opt. 1991. V. 38. N 11. P. 2323. doi 10.1080/09500349114552371

[12] Zharikov E.V., Lis D.A., Subbotin K.A., Dudnikova V.B., Zaitseva O.N. // Phys. Pol. A. 2013. V. 124. N 2. P. 274. doi 10.12693/APhysPolA.124.274

[13] Akgul F.A., Gumu C., Er A.O., Farha A.H., Akgul G., Ufuktepe Y., Liu Z. // J. Alloys Compd. 2013. V. 579. P. 50. doi 10.1016/j.jallcom.2013.05.057

[14] Марфунин А.С. Введение в физику минералов. М.: Недра, 1974. $328 \mathrm{c}$

[15] Dmitruk M.V., Kaminskii A.A. // Soviet Physics JETP. 1968. V. 26. N 3. P. 531.

[16] Denks V., Savikhina T., Nagirnyi V. // Appl. Surf. Sci. 2000. V. 158. N 3. P. 301. doi 10.1016/S0169-4332(00)00009-X

[17] Williams R.T., Nagel D.J., Klein P.H., Weber M.J. // J. Appl. Phys. 1981. V. 52. N 10. P. 6279. doi 10.1063/1.328572

[18] Soifer L.M., Shakhnovich M.I., Chubenko A.I., Blank A.B. // J. Appl. Spect. 1965. V. 2. N 1. P. 15. doi 10.1007/BF00658077

[19] Bezhanov V.A., Mikhailin V.V., Chernov S.P., Karimov D.N., Sobolev B.P. // Opt. Spectrosc. 2006. V. 101. N 4. P. 571. doi 10.1134/S0030400X06100122

[20] Xue C., Yi K., Wei C., Shao J., Fan Z. // Proc. SPIE. 2009. V. 7283. P. 72831E. doi 10.1117/12.828661

[21] Guyot Y., Collombet A., Somatri T., Tkachuk A., Joubert M.-F. // J. Alloys Compd. 2002. V. 341. P. 174-178.

[22] Masada I., Nawata T., Inui Y., Date T., Mabuchi T., Nishijima E., Fukuda T. // Proc. SPIE. 2006. V. 6154. P. 61542P. doi 10.1117/12.657249 